\title{
MEMORY PROPERTIES OF NON-FULLY INTERCONNECTED NEURAL NETWORK*
}

\author{
R.A. KosińSKI AND $\Lambda$. ZAGórSKI \\ Dept. of Applied Pliysics and Mathematics, Institute of Physics \\ Warsaw University of Techuology, Koszykowa 75, 00-662 Warszawa, Poland \\ (Reccived February 4, 1994; revised version May 13, 1994)

\begin{abstract}
A model of a neural network consisting of two states neurons with the number $K$ of (symmetric) synaptic connections per neuron treated as a variable was investigated uumerically. Ilebb's rule was used for storing uncorrelated patterns in the network. A maximal number of such patterns, which can be effectively retrieved by the network and the process of deterioration of the memory, is examined as a function of the number of synaptic connections per neuron. The influence of the number of neurons in the network as well as boundary conditions for the storage capacity of the network are discussed.
\end{abstract}

PACS numbers: $87.10 .+\mathrm{e}$

\section{Introduction}

A ncural network is a nonlinear dynamical system which provides a simple model of a content-addressable memory. After a process of learning a number of random patterns can be stored in a network - they compose a set of attractors of a dynamics of the network. In the Ilopficld model [1] a formal neural network consists of a set of neurons having two possible states $S_{i}=+1$ (firing) and $S_{i}=-1$ (rest). In this model all neurons are fully interconnected. Assuming zero temperature limit, which means that the stochastic eflects in the ncuron dynamics are absent, the state $S_{i}(t)$ of an $i$-th neuron at the time $t$ is defined by the local field $h_{i}$ acting on this ncuron and coming from other ncurons at the time $(l-1)$

$$
S_{i}(l)=\operatorname{sign}\left[h_{i}(l)\right],
$$

where

$$
h_{i}(t)=\sum_{j=1}^{\mathrm{N}} J_{i j} S_{j}(l-1)
$$

*This work was supported by the Grant No. 223219102 of the State Cornmiltee for Scientific Research (Republic of Poland). 
and $N$ is the number of neurons in the network. $J_{i j}$, called synaptic connections, characterize couplings between neurons $i$-th and $j$-th and, in general, can be symmetric or antisymmetric. They have the form depending on the patterns which were stored in the neural networks during the process of learning. Sevcral learning rules were proposed in the literature [2-7]. One of the most extensively investigated is the onc proposed by IIebb [2], to which a number of modifications investigated both analytically and numerically have been published [3-9]. In this rule, during the process of learning of a certain pattern $\left\{\mu_{i}^{\alpha}\right\}$, the synaptic connections between the $i$-th and $j$-th neuron are modified in the following way:

$$
\Delta J i j=\frac{1}{N} \mu_{i}^{\alpha} \mu_{j}^{\alpha}
$$

(most commonly, as a starting neural configuration the state of labula rasa, i.e. with all $J_{i j}=0$, is assumed [5]). As a consequence of the successive learning of $M$ patterns the synaptic connections have the form

$$
J_{i j}=\frac{1}{N} \sum_{\alpha=1}^{M} \mu_{i}^{\alpha} \mu_{j}^{\alpha} \text { with } J_{i i}=0 .
$$

As results from Eq. (1) connections between neurons are symmetric, this enables one to adopt the methods of statistical mechanics developed for a spin glass $[3-5,8-11]$. In particular, the energy function can be introduced, which for $T=0$ decreases during the time evolution of the network.

\section{The model}

In the present work we numerically investigate a modification of the Ilopfield model with a certain type of dilution appcaring in the dynamic cvolution of the network. The learning rule of standard IIcbb's form given by Eqs. $(3,4)$ is used, i.e. the synaptic connections are created for cach pair of neurons as results from the form of the patterns stored in the network. We assume, however, that in the time evolution of the network not all synaptic connections are active: effective connections of the $i$-th neuron are possible only with the neurons having indices $i-K<i<i+K$, provided $i-K \geq 1$ and $i+K \leq N$. Thus, the neurons with the indices near to $i=1$ and $i=N$ have a smaller number of synaptic connections than others and, for the introduced relation of order of the set of neurons, they may be treated as neurons located close to the physical boundarics of the network (below they will be called boundary neurons). Such an assumption may reflect various processes which reduce the influence of some regions of the network on the neuron under consideration. Thlis may correspond to the situation in which some synaptic connections in the network have become inactive or damaged.

The difference between our and other models of diluted networks [7, 11-15] may be described as follows. In our model different neurons lave a different (but constant) number of connections - boundary ncurons lave a smaller number of connections. Morcover, the dilution of synaptic comnections appears in the process of dynamic evolution of the nchwork and it has a deterministic character. On the other hand, in the other diluted networks the average number of synaptic connections is the same for each neuron - all of them are treated homogencously. 
The stochastic factor appears already in the process of learning and relates to each pair of neurons. During the time evolution of such networks all existing connections are active. Thus, a part of information about memorized patterns is lost at the very beginning.

In our model the local field acting on the $i$-th neuron and coming from the $2 K-1$ other neurons is given by

$$
h_{i}(l)=\sum_{j=i-K}^{i+K} J_{i j} S_{j}(l-1),
$$

$\sum^{\prime}$ means that the terms with $i-K<1$ or $i+K>N$ are excluded.

It should be noted that for small valucs of $K$ the influence of the boundary neurons on the dynamic properties of the network is negligible. With the increasing $K$ this influence increases. Starting from $K=0.5 N$ neurons, which are fully connected to other neurons, appear in the network. For $K=N$ (see Eq. (5)) our model agrees with the standard Ilopficld model [1]. In this case the difference between neurons inside the network and those lying at the boundary of the network disappears. 'Thus, the most distinct influence of the used boundary conditions on its dynamical properties should appear for $1 \ll K \ll N$.

For our model a storage capacity of the network is analysed. The maximal number of stored patterns $N_{+}$which have a sufficicutly large overlap with the attractors of the network as a function of $K$ is found numerically for the networks with $N=100,400$, and 700 neurons. The process of deterioration of the memory after exceeding $N_{+}$as a function of $K$ is investigated also for the networks with different $N$.

Additionally, a modification of the previous model, in which the local field (Eq. (5)) is calculated with the randomly chosen number of connections $K$ per each neuron and constant during each time cvolution of the network, is investigated numerically. In this case, for each value of $K$ every neuron is connected with its nearest neighbours. This is not true for standard diluted models [7, 11-15] (where neurons having connections only wilh the remote neighbours may occur), irrespectively of the ordering of neurons introduced in the network.

\section{Numerical results}

In our computations a specificd number $N_{\mathrm{p}}$ of uncorrelated random patterns was stored at a time $\ell=0$ in the network using the IIebb learning rule given by Eq. (4). Next, starting from an initial neuron configuration, the states of all neurons at a time $t+1$ were synchronously upelated according to the local field coming from neurons (situated in a spliere of a radius $K$ ) at a time $l$ (sce Eq. (5)). In order to control. whether an $i$-lh pattern can be eflectively retrieved, time evolution of the network was computed till the network reached its altractor. This procedure was repeated for 100 different initial configurations, which differ from the $i$-th pattern in the state of one, randomly located, neuron. It was assumed that, if in 95 cases the time evolution of the network results in the configuration in which states of less than $0.05 \mathrm{~N}$ ncurons did not agree with the $i$-th pattern, this pattern was effectively retrieved by the network. (The overlap of the current network state 
with the $i$-th pattern was controlled after cvery 10 time steps). The choice of the initial configuration described above secured that we did not leave the basin of attraction of the $i$-th pattern. In the number of cases it was checked, however, that deflections of more than one (up to 10) randomly chosen neurons from its state corresponding to the $i$-th pattern did not change the results of computation.

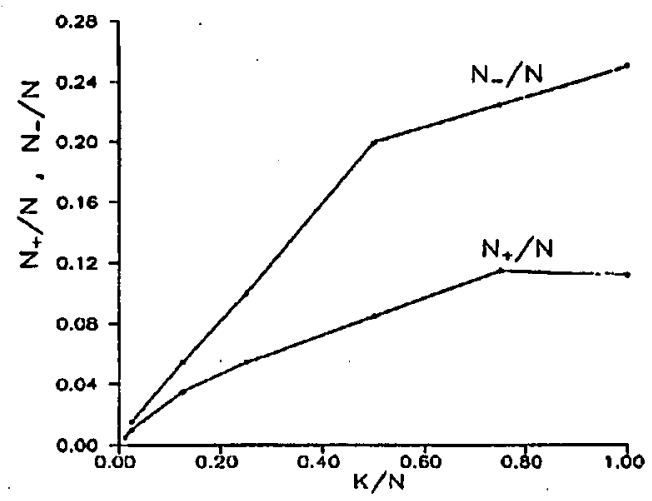

Fig. 1. Storage capacity of the network of $N=400$ neurons as a function of the relative number of synaptic connections per neuron $K / N . N_{+} / N$ is the maximal number of stored patterns which can be effectively retricved by a network; $N_{-} / N$ is the maximal number of stored patterns for which at least one pattern can be effectively retrieved by the network.

In Fig. 1 the ratio of effectively retrieved palterns to the total number of neurons $N_{+} / N$ as a function of number of synaptic connections per neuron $K / N$ is shown for $N=400$. It can be seen that $N_{+} / N$ increases with the number of connections per neuron. Starting from $K / N=0.5$ neurons fully connected to others appear in the network. For $K / N>0.75$ the saturation of the curve is observed which means that almost all neurons are fully connected, except only a small number of boundary neurons. This means that the increase in the synaptic connections per neuron above about $0.75 \mathrm{~N}$ does not result in the increase in the storage capacity of the network. Similar saturation of storage capacity for $K \cong N$ was observed also for the standard diluted networks [7, 11-15]. For the fully connected network $(K / N=1)$ the ratio $N_{+} / N$ equals about 0.12 , which is close to the critical value $\alpha_{c} \equiv N_{+} / N=0.138$ obtained for the Ilopfield model [1]. The difference is connected with the inhomogeneity of our network and - independently - with its finite size $[11,16]$.

As a result from our computations, after exceeding a critical storage value $\alpha_{c}$, the blackout catastrophe, as was found for the IIopfield model, is not observed. The patterns are forgotten gradually, what shows the curve $N_{-} / N$ in Fig. 1. Here,

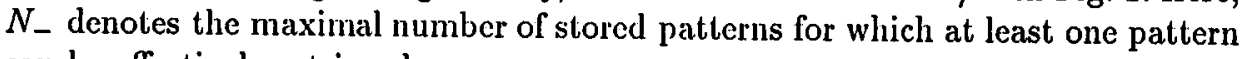
can be effectively retrieved.

This result is better visualised in Fig. 2 (curve a), which is plotted for $N=400$ and for a fully connected network $(K=400)$. In this figure $N_{\mathrm{p}}$ de- 


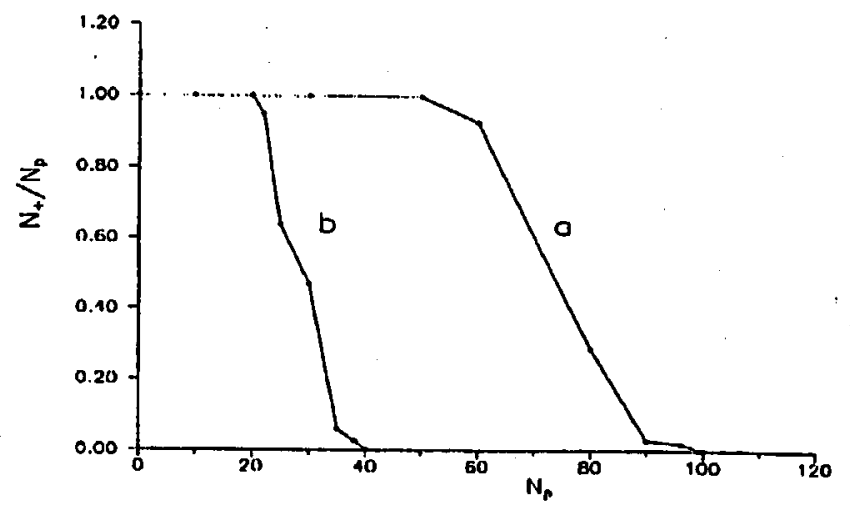

Fig. 2. The process of deterioration of the memory of the network of $N=400$ neurons. $N_{\mathrm{p}}$ is the number of stored patterns, $N_{+} / N$ is the relative number of patterns which can be effectively retrieved by the network. Curve a - fully connected network $(K=N)$. Curve $b$ - random number of synaptic connections per neuron during the time evolution of the network.

notes the number of stored patterns in the network, while $N_{+}$is, as previously, the number of effectively retrieved patterns. It can be seen that for $N_{\mathrm{p}}<50,95 \%$ memorized patterns can be effectively retrieved. For $N_{\mathrm{p}}>50$ however, the number $N_{+}$decreases and for $N_{\mathrm{p}}>100$ none pattern can be retrieved by the network in its time evolution. Thus, the total confusion of the network (i.e. the situation in which none of the stored patterns is an attractor of the network) occurs at $N_{\mathrm{p}} / N=0.25$, which is approximately two times larger than $N_{+} / N$. Note the tail of the discussed curve which occurs near $N=100$. It means that single patterns may be effectively retrieved even when the total number of stored patterns in the network is relatively high. Similar behaviour was found earlier $[11,16]$, where also the retrievals of patterns wilh overlaps much less than 1 were analysed.

A comparison of the storage capacity of networks of different sizes is shown in Fig. 3 for $N=100$ (curve a), $N=400$ (curve b) and $N=700$ (curve c) neurons. As previously, $N_{+} / N$ denotes the ratio of effectively retrieved patterns to the total number of neurons and $K / N$ denotes the number of synaptic connections per neuron. For the smaller network (with $N=100$ neurons - curve a) the influence of boundary neurons on the dynamical properties of the network is greater than for networks with $N=400$ and 700 . Therefore, the relation $N_{+} / N$ vs. $K / N$ for $N=100$ increases and does not indicate saturation like curves $b$ and $c$ (for $N=400$ and 700 , respectively). For such networks also greater memory effectiveness than for the larger ones is observed (c.f. location of curve a in comparison with the location of curves $b$ and $c$ ). On the other hand, the curves $b$ and $c$ are located close to each other. This indicates that the influence of the boundary neurons on the dynamical properties of the network decays and means that further increasing of the network's size does not influence essentially the relative storage capacity of the network. The saturation which is seen on these curves for $K / N>0.75$ indicates that almost all neurons are fully interconnected and further increasing of 


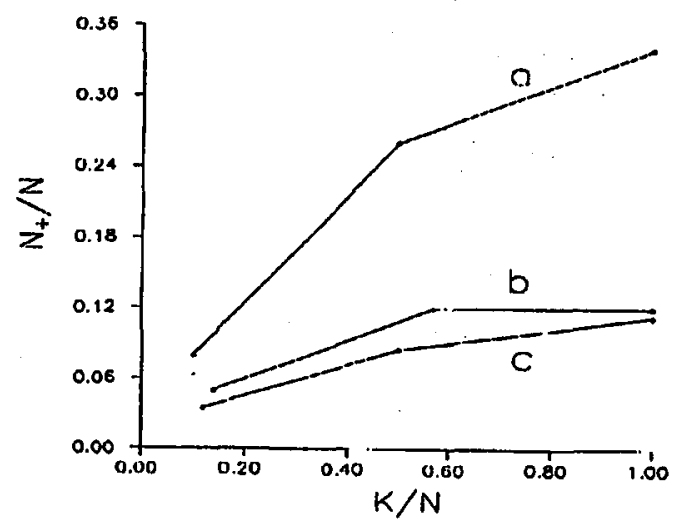

Fig. 3. Storage capacity of the network consisting of $N=100$ ncurons - curve a; $N=400$ neurons - curve b and $N=700$ neurons - curve c. $N_{+}$and $K$ have the same meaning as in Fig. 1.

$K$ does not improve memory capacity of the network. The observed dependence of $N_{+} / N$ vs. $K / N$ on the number of neurons in the network is influenced also by the size effect discussed by many authors (see Amit's book [11] and references therein). This effect seems to be responsible for the fact that, for $K \cong N$, the curve a lies higher than the curves $b$ and $c$.

In a part of numerical computations random numbers of synaptic connections per neuron in the time evolution of the network $N=400$ was assumed. An interesting result concerns the process of deterioration of the memory. It is presented on the curve $b$ in Fig 2 . It can be seen that the process of deterioration begins earlier than for the case of a fully connected network (curve a) and proceeds faster. The same characteristic tail as in the curve a occurs near $N_{\mathrm{p}}=40$.

\section{Conclusions}

In the present work a modification of the Ilopfield model of neural networks in which the number $K$ of the synaptic connections per neuron is treated as a variable, is analysed numerically. Some of the neurons, located at the boundary of the network, have less synaptic connections than those located inside the work. The influence of these boundary neurons on the dynamic properties of the network is significant for $1 \ll K \ll N$ and is more important for smaller networks. It was found that the number of synaptic connections per neuron $K$ has a significant influence on the storage capacity of neural networks. It was observed also that the process of deterioration of the memory due to its overloading does not occur abruptly, as for the IIopfield model, but is gradual. This scems to be connected with the limited size of the networks and the calculations with the greater number $N$ would be interesting. 


\section{References}

[1] J. Ilopficld, Proc. Nall. Acad. Sci. (USA) 79, 2554 (1982).

[2] D.O. IIebb, The Organizalion of Behaviour, Wiley, New York 1949.

[3] G. Parisi, J. Phys. A, Malh. Gen. 10, L675 (1986).

[4] M. Mezard, J.P. Naclal, G. Toulouse, J. Plhys. (Paris) 47, 1457 (1986).

[5] G. Toulouse, S. Dehacue, J.-P. Clangeux, Proc. Nall. Acad. Sci. (USA) 83, 1695 (1986).

[6] J.P. Nadal, G. Toulouse, J.-P. Cliangeux, S. Deliacnc, Europhys. Lelt. 1, 535 (1986).

[7] P. Spitzner, W. Küinzcl, Z. Plyys. B 74, 539 (1989).

[8] D.J. Amit, II. Gut freund, II. Sompolinsky, Phys. Rev. A 32, 1007 (1985).

[9] D.J. Amit, II. Gutfreund, II. Sompolinsky, Phys. Rev. Lett. 55, 1530 (1985).

[10] E. Gardner, B. Derrida, J. Phys. A, Malh. Gen. 21, 271 (1988).

[11] D.J. Amit, Mfodeling Brain Funclions, Cambridge University Press, New York 1989.

[12] II. Sompolinsky, Plyys. Rev. A 34, 2571 (1986).

[13] T.L.II. Watkin, D. Sherrington, Europhys. Lell. 14, 791 (1991).

[14] A.E. Patrick, V.A. Zgrebuow, J. Phys. A, Math. Gicn. 24, 3413 (1991).

[15] B. Derrida, Phys. Scr. Vol. T 29, 24 (1989).

[16] D.J. Amit, II. Gut freund, II. Sompolinsky, Ann. Phys. 173, 30 (1987). 